\title{
Construir la ciudad desde los espacios públicos: herramientas de análisis y evaluación de tipologías urbanas para la integración socio-espacial
}

Building the city from public space: Analysis and evaluation tools for the assessment of sociospatial integration promoting urban typologies

Mónica Sánchez Sepúlveda

\section{Filiación}

Pontificia Universidad Católica de Puerto Rico

E mail: sanchezmonicav@gmail.com

Primera versión recibida en: 05 de diciembre, 2014

Última versión recibida en: 28 de junio, 2015

\section{Resumen}

Ante el déficit de espacio público como factor generador de calidad de vida urbana en términos de desaprovechamiento de las oportunidades del territorio, pérdida de identidad y fraccionamiento social, se plantea una metodología de análisis y evaluación urbana de los espacios públicos basada en datos cualitativos, con una base teórica, para que pueda ser utilizada en la práctica urbanística. El manuscrito se centra en el potencial de la proyección del espacio urbano percibido y jerarquías espaciales como consecuencia de los usos sociales junto con los cambios espacio-temporales como el crecimiento demográfico y la desintegración social. Para ello, se revisan diversas nociones del espacio público y elementos de correspondencia en la ciudad de Santiago de Chile. Con vistas a comprender el comportamiento y funcionalidad del espacio público, se elabora una metodología para la clasificación de los espacios públicos sobre las dimensiones: morfo-tipológica, funcional y socio-psicológica a partir de las relaciones que se establecen entre el hombre y su medio. Se concluye la importancia de develar estos discursos para añadir un nuevo nivel para el modo eficaz de las infraestructuras urbanas según sus necesidades y condiciones - es decir, el nivel de los efectos socioespaciales.

\section{Palabras clave}

Espacios públicos, tipología, integración social, convivencia urbana, convergencias, alteridad

\begin{abstract}
With the lack of public space as a factor that generates urban quality of life in terms of missed opportunities of the territory, loss of identity and social division, is proposed a methodology for analysis and evaluation of the urban public space based on qualitative data, with a theoretical basis, so it can be used in planning practice. The manuscript is centered on the potential projection of perceived urban space and spatial hierarchies as a result of the social uses along with temporal-spatial changes such as population growth and social disintegration. For this, it is reviewed various notions of public space and elements of correspondence in the city of Santiago, Chile. In order to understand the behavior and function of public space, a methodology for classification of public spaces is made on the dimensions: morpho-typology, functional and socio-psychological from the relationships established between man and his environment. It is concluded that uncovering these discourses becomes fundamental to add a new level to effective urban infrastructure according to their needs and conditions, significantly increasing the socio-spatial impacts.
\end{abstract}

Keywords

Public spaces, typology, social inclusion, urban living, convergences, otherness 


\section{Sumario}

Introducción

1 Espacio público: la planificación y la espontaneidad

2 Alteridad y Cultura Urbana

3 Fundamentos e Hipótesis

4 Técnicas de Investigación

\subsection{FASE 1: Estudio Epistemológico - Perspectiva Humana}

4.2 FASE 2 Estudios cuantitativos - Configuraciones de espacios públicos

\section{Conclusión}

\section{Referencias}

\section{Introducción}

La ciudad es una es una realidad en continuo movimiento sometida a parámetros espaciotemporales, necesidades culturales, sociales y económicas. Alicia Lindón dice que "las metodologías urbanas tradicionales observan el espacio urbano desde afuera de la experiencia espacial, desde afuera del sujeto habitante. Por eso suelen tratar al espacio urbano como objeto" (Lindón, 2007; p. 39). A pesar de que la ciudad es una construcción material, también es una experiencia mental condicionada dentro de esos mismos parámetros espaciotemporales. Desde una perspectiva visual y morfológica se puede distinguir una ciudad. La ciudad como realidad objetiva y como imagen simbolizada cumple un papel fundamental en la organización del espacio. No obstante, el fenómeno urbano que completa la estructura urbana es su representación como un producto social, resultado de la acción humana.

El espacio público entendido como el escenario para la dispersión de la ciudadanía no puede ser separado del tipo de vínculo social deseado, tipos de estilos de vida necesarios y el tipo de organización espacial demandada. Una forma de acercarse a las formas de vida en la ciudad, es investigar el mosaico social urbano. Las ciudades son agregaciones de individuos que se integran en unidades de diferente amplitud, como casas, calles, lugares de trabajo y espacios públicos. Precisamente los espacios públicos son fundamentales para la comprensión de la estructura urbana. Este artículo estudia los umbrales de tolerancia que establecen las clases sociales que viven con otra diferente, en diversos espacios públicos que estructuran la vida de la ciudad. Para esta investigación se estudia el caso de Santiago, Chile, donde la estructura social es un elemento destacado.

Observando la rutina del día a día y los actos multitudinarios intermitentes que mantienen la dinámica de la ciudad, analizándolas tipologías de los espacios públicos y con base de estudios disponibles este trabajo se pretende descubrir que pasa con el espacio, su relación con las personas que frecuentan el lugar y las situaciones que allí se generan. Se concluye que las distinciones que las personas realizan no están del todo alineadas a los ingresos sino por elementos transversales como el esfuerzo, el territorio, etc. En la práctica la gente experimenta mayor diversidad socioeconómica de la que pude reconocer. El mayor factor de distinción son socioculturales y no necesariamente económicos, se abren posibilidades de mezcla espacial en términos socioeconómicos.

\section{Espacio público: la planificación y la espontaneidad}

La comprensión de lo que es el espacio público tiene varios significados. Por un lado, están los que definen que lo "público" se refiere a lo que es de interés o de uso común, en lugar de 
"privado" que representaría el interés y el uso individual (Arendt, 1993; Habermas 1994; Sennett, 1977). Mientras que hay quienes lo ven como una espacialidad definida materialmente (Caldeira, 2000; Goodsell, 2003). Mientras que el primer sentido se relaciona con el interés y el bien común, la segunda es para reclamar la existencia de una superficie material y simbólica.

A diferencia del espacio público en Europa, en América Latina en general y, en Chile en particular, el Estado tiene un papel clave en la configuración del espacio público. Entre la dictadura (1973-1990) y la transición (1990-1994), la sociedad chilena experimentó el movimiento de los cambios culturales. Siendo esto factor de transformación del espacio público y afectando directamente el objetivo y la experiencia subjetiva de la sociedad. Desde hace algún tiempo el espacio público ha adquirido una nueva importancia y se instaló en el centro del debate contemporáneo en diversos campos como la sociología, la economía y la planificación urbana. Es este mismo contexto, políticos y académicos coinciden en que la recuperación de los espacios públicos debe ser la principal prioridad estratégica del desarrollo urbano en la próxima década. Sin embargo, algunos distritos financieros en Santiago de Chile son criticados por la generación de espacios públicos de baja calidad, dando prioridad a los intereses privados antes que los de la comunidad. Mediante el desarrollo de los edificios de forma individual, la posibilidad de un espacio público unitario se pierde, por lo que terminan siendo espacios desérticos entre edificios. Si el espacio público generara beneficios a los inversores y a los futuros propietarios, podrían cambiar la actitud hacia el desarrollo de éstos elevando los programas y proyectos que se desarrollan y abriendo pensamiento a la forma en que entiende las líneas de política pública del espacio público que abordarlos.

\section{Alteridad y Cultura Urbana}

¿Cuáles son las oportunidades de contacto social actualmente generadas dentro de este entorno urbano, llamado espacio público? ¿Cómo se valoran estas oportunidades? Para evaluar el nivel de contacto con uno diferente, es necesario entender la imagen que cada clase social tiene de la otra. Parece evidente que las desigualdades sociales son un elemento importante en la estructura social chilena. Al analizar los distintos sectores de Santiago de Chile de acuerdo a las configuraciones de los espacios públicos, se observan procesos de segregación claramente espaciales; esta es la brecha entre diferentes clases socioeconómicas. (Mapa 1) En las últimas décadas, la sociedad chilena ha experimentado profundos cambios en sus características estructurales, que ha definido de diversas formas el contacto entre personas de diferentes niveles socioeconómicos y, por lo tanto, en las formas de cohesión social (Hopenhayn, 2007). Con el crecimiento demográfico de Santiago diferentes grupos socioeconómicos están aislados entre sí produciendo guetos visibles. Un ejemplo de estos cambios es la expansión y la movilidad de la clase media (Gráfico 1) sobre todo en el ámbito urbano (Torche y Wormald, 2004), la cual se ha traducido en un aumento de la diversidad social.

La movilidad social ha sido importante en Chile en las últimas décadas en la medida en que no ha habido crecimiento económico y reducción de la pobreza. El MIDEPLAN, CASEN 1990-2009 describe como en 1990 los niveles de pobreza alcanzaban al 38.6\% de la población, el año 2006 dicha proporción se había reducido al 13.7\%. No obstante, en la medición del año 2009 se produjo un leve aumento: la cifra fue del $15.1 \%$. Con ello, el Coeficiente Gini pone de manifiesto haberse estancado el número de pobres (Barozet y Fierro). Varios autores sostienen que este tipo de decrecimiento, especialmente la pobreza, tiene un impacto negativo en varias áreas: trabajo (Wilson, 1997; Sabatini et al, 2008), educación (Jencks y Mayer, 1990; Flores, 2008), desarrollo social y emocional (Brooks-Gunn, 1992), entre otros. El argumento central de esta parte de la investigación es que el contacto social entre las diferentes clases se beneficiaría no sólo individualmente, sino también puede ser fundamental para la generación de un comportamiento coherente. Por lo tanto, bajo ciertas condiciones, un mayor grado de 
proximidad espacial y el contacto social entre personas de diferentes niveles socioeconómicos, fomenta la generación de normas y valores que generen actitudes y comportamientos que conduzcan a la supervivencia y el desarrollo de una sociedad. ¿Cómo los espacios públicos afectan la experiencia del contacto entre personas de diferentes clases sociales? (Fig. 1)

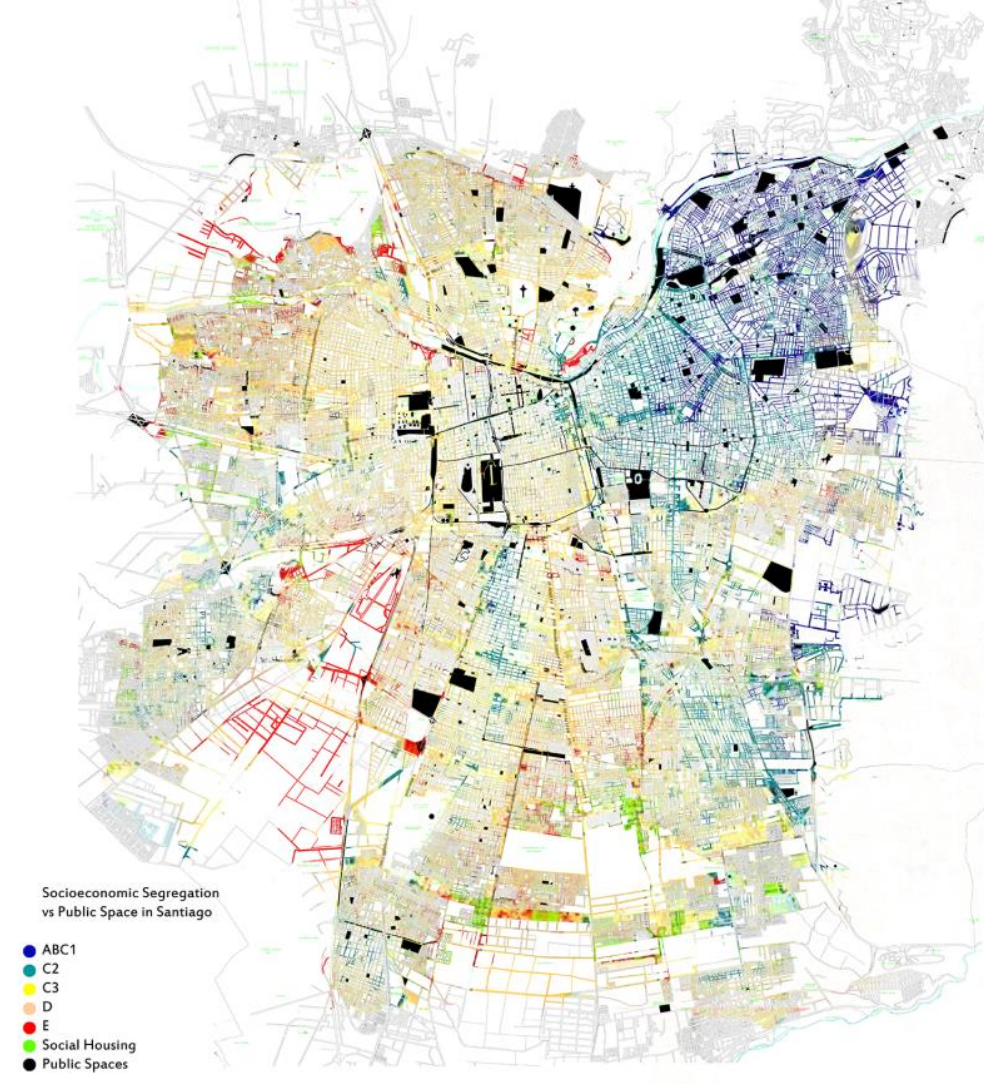

Mapa 1 Segregación socioeconómica vs. espacio público en Santiago de Chile Fte.: Elaboración propia

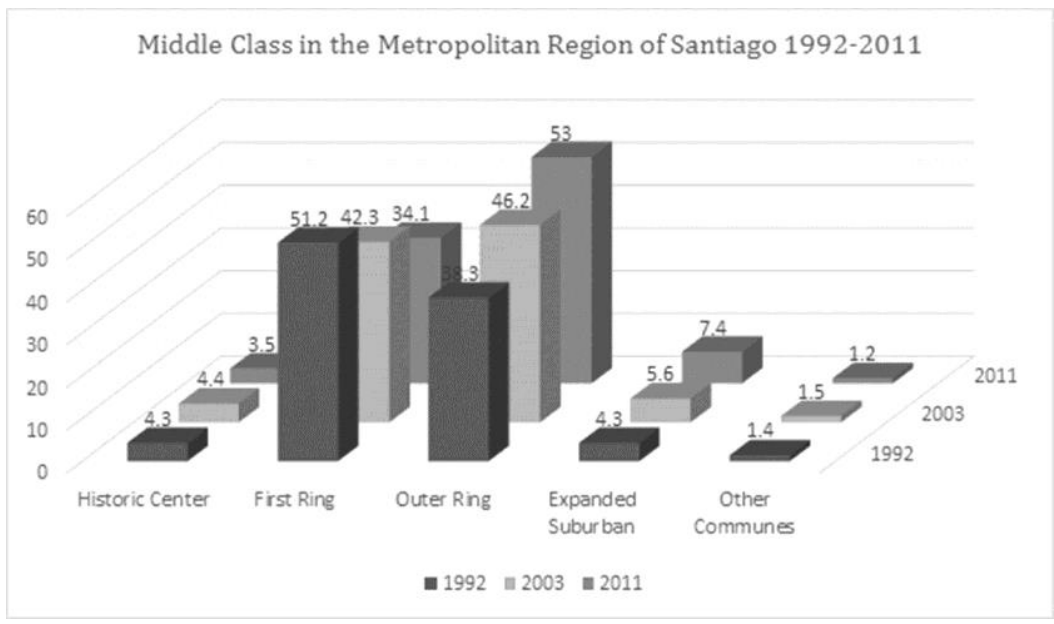

Gráfico 1 Clase media en la Región Metropolitana de Santiago 1992 - 2011 Fte. Elaboración propia 


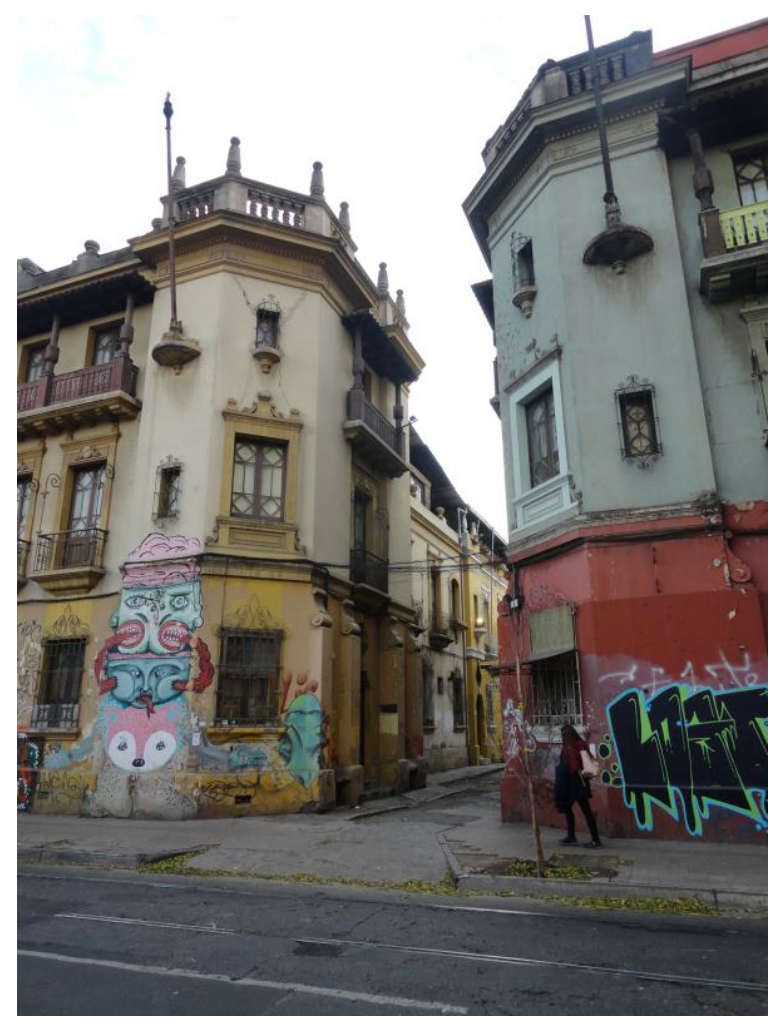

Fig. 1 Santiago de Chile

Fte. Elaboración propia

\section{Fundamentos e Hipótesis}

La ciencia de la equística, liderada por Konstantinos Apostolos Doxiadis, sirve como un método de análisis y solución de los fenómenos y problemas que surgen allí donde se establece el hombre. En contraste con la arquitectura o enfoque urbano, la equística es una ciencia cuya tarea es examinar los asentamientos humanos desde todos los ángulos posibles para lograr el desarrollo de soluciones técnicas a sus problemas inherentes. Uno de los principios de equística explica que el hombre trata de aumentar sus posibilidades de contacto con su entorno físico y natural con sus compañeros y otros asentamientos (haciendo un mejor uso de sus redes), tratando de minimizar el esfuerzo necesario durante las actividades se llevan a cabo todos los días en la forma más fácil posible. Distinto a temas vinculados a los ingresos, sino por miembros transversales tales como el esfuerzo y oportunidades.

Una aproximación cualitativa a habitantes de diferentes orígenes sociales pertenecientes a la Región Metropolitana de Santiago sugiere una heterogeneidad que se produce sobre todo en los espacios públicos. Si los espacios públicos actuales de la ciudad de Santiago pueden estar generando más segregación de la que la gente realmente percibe, afectando a la tolerancia de contactos con personas de diferentes clases sociales en diferentes tipos de espacio, entonces las estructuras son un catalítico que afecta a la cultura (la estructura física puede estar modulando la relación entre diferentes). Una sociedad que ofrece oportunidades de contacto más altos y valiosos entre los diferentes grupos mostraría una tendencia a ser más cohesiva en un contexto menos conflictivo, siempre y cuando la gente adopte este tipo de oportunidades. Esta hipótesis, se basa en los datos de observación en la determinación del comportamiento en la integración. 


\section{Técnicas de Investigación}

Para desarrollar esta investigación se comenzó por comprender cómo las clases sociales se acercan al diferente, observado desde la perspectiva de los que están en tránsito, cómo se ha transformado hasta tu estado actual, analizando la historia, las características sociales y físicas de la ciudad de Santiago. Por medio de la observación y diario de campo se analizaron las formas de contacto y las oportunidades espaciales para estos contactos. A su misma vez, realizando estudios cuantitativos que representan diferentes características de escala, la distribución de las clases sociales, la penetración de la privada / pública de servicios, redes, la apertura, servicios, etc. se busca entender los aspectos tangibles y evidentes de los espacios públicos. Esto, nos lleva a categorizar las tipologías de espacios públicos por parte de la función, el tamaño y las características de diseño predominantes. La intangibilidad se investiga entonces a través de entrevistas cualitativas a poblaciones particulares de diferentes clases sociales (la movilidad, los valores culturales, la apertura, la voluntad, los elementos que influyen en las posibilidades de contacto, etc.).

\subsection{FASE 1: Estudio Epistemológico - Perspectiva Humana}

La cohesión social entendida como la voluntad de los individuos para construir patrones de vivir con "otro" de distinta condición social y construido a partir de una convivencia imaginaria favorable (Márquez, 2007), depende de dos dimensiones. Una es la subjetiva, que se refiere a las valoraciones culturales de la integración, la experiencia y los vínculos sociales; y la otra es objetiva y se relaciona con la mezcla y la experiencia de oportunidades con otro. Bajo estas dimensiones se estudió: 1) "Disposición de un urbana la Integración Social en Santiago, Antofagasta y Temuco: Una mirada desde la Integración residencial" (Estudio DIS) realizado en 2008 por ProUrbana-PUC y Observatorio Social-Universidad Alberto Hurtado (UAH), Ministerio de Vivienda y Urbanismo de Chile, donde se entrevistaron a 1,049 personas de Gran Santiago en el 2008; 2) entrevistas realizadas a grupos socioeconómicos diferentes en la ciudad de Santiago, en las comunas de: Las Condes, Providencia, Santiago Centro, San Joaquín, Quinta Normal, Estación Central, Recoleta y Maipú, a un total de 39 personas en la forma de una conversación abierta en el 2014, basada en temas predefinidos en un patrón temático que era: la experiencia del contacto con personas de otra clase en diferentes espacios y la inclinación a ponerse en contacto con otra diferente clase social - entrevistados, independientemente de la edad, el nivel socioeconómico o el sexo. El análisis de este estudio de caso y estudio de fuente secundarias, determinaron las experiencias reales de contacto entre personas de diferente clase social, el significado dado a esas experiencias y la disposición a tener más contacto con otras personas de diferentes clases sociales.

Se desprende que por una parte, la proximidad se atribuye una serie de beneficios funcionales para las personas con menos recursos, tales como una mejor infraestructura urbana, espacios públicos y equipamientos (Gráfico 2). Los encuestados eran entusiastas acerca de la integración pero, cuando se les pregunta si la mayoría de la gente piensa así, los niveles de acuerdo eran menos. Por esta razón, es importante entender la visión que tienen de lo que otros piensan sobre el mismo tema. Curiosamente, a pesar de que son inferiores a las declaradas por sí mismos, esto revela la existencia de espacio para pensar las políticas de pro integración (Gráfico 3). También es interesante comprobar lo que la existencia de la deseabilidad social en esta respuesta que revela que estar a favor de la integración social es valorado socialmente y que, por lo tanto, se es negativo el cierre de esa posibilidad.

Lo anterior permite entender que el discurso que rodea la integración social es favorable. Sin embargo, hay elementos que dificultan a la gente que se traslada a vivir a sectores socialmente mezclados. Si la mezcla es posible mediante la instalación de algunas condiciones básicas, entonces es posible concluir que los elementos que dificultan la prestación a la mezcla no son 
cultura, pero en las circunstancias específicas en que se produce. Según el Estudio DIS el mayor por ciento preferiría vivir cerca de los lugares que frecuentan y con espacios de recreación (Gráfico 4), el menor porcentaje es la composición social del barrio. En este sentido, muchas personas probablemente dispuestas a vivir con personas de otros estratos sociales si la ubicación ofrece otros atributos que parecen importantes para ellos. Además, a pesar de que la mayoría de los encuestados respondió que era bueno que las familias de diferentes clases sociales vivan cerca, dos tercios de ellos prefieren vivir en un barrio con familias de su propia clase y sólo el 17 por ciento dicen que les gustaría si su barrio también incorporan las familias de otras clases sociales (Gráfico 5 ).

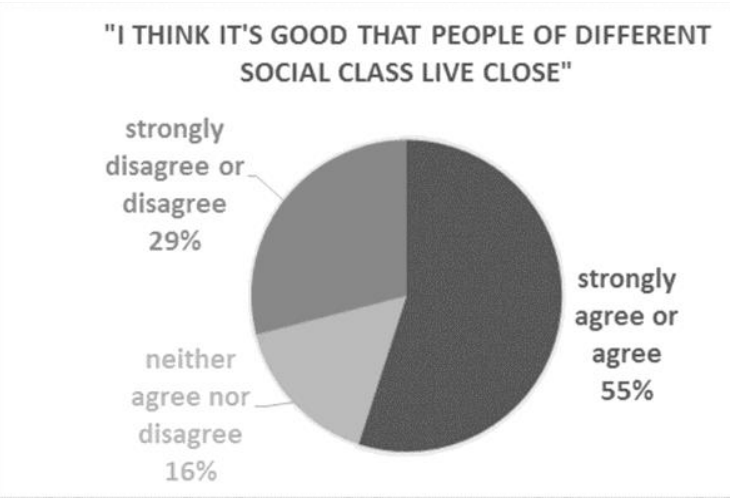

Gráfico 2 Preferencias respecto de mixtura social Fte.: Elaboración propia

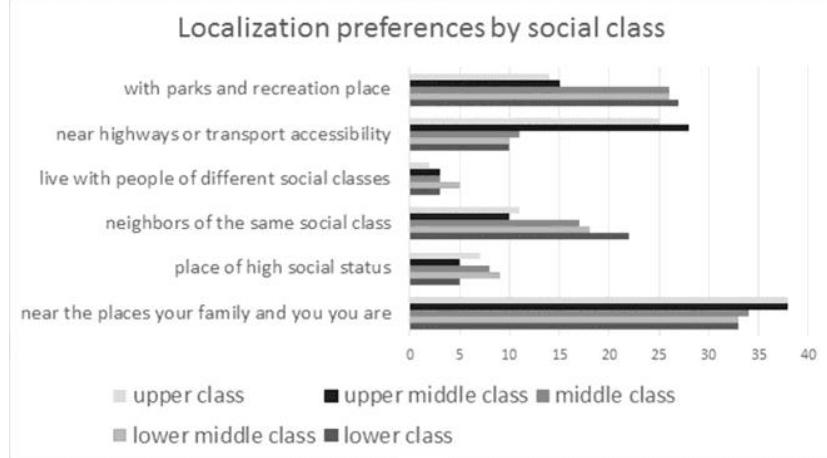

Gráfico 4 Preferencias de localización según clase social

Fte.: Elaboración propia
"MOST PEOPLE THINK IT'S GOOD THAT PEOPLE OF DIFFERENT SOCIAL CLASS LIVE CLOSE"

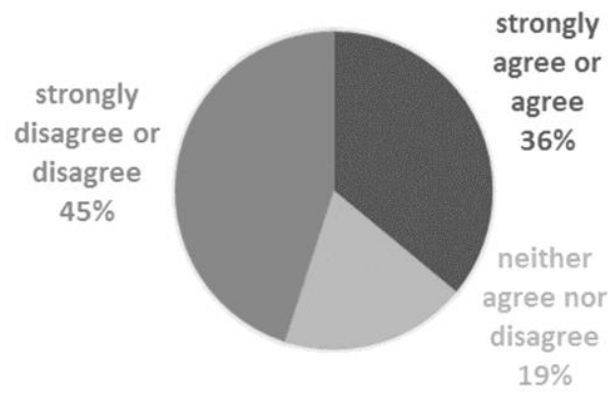

Gráfico 3 Percepción de preferencias respecto de mixtura social

Fte.: Elaboración propia

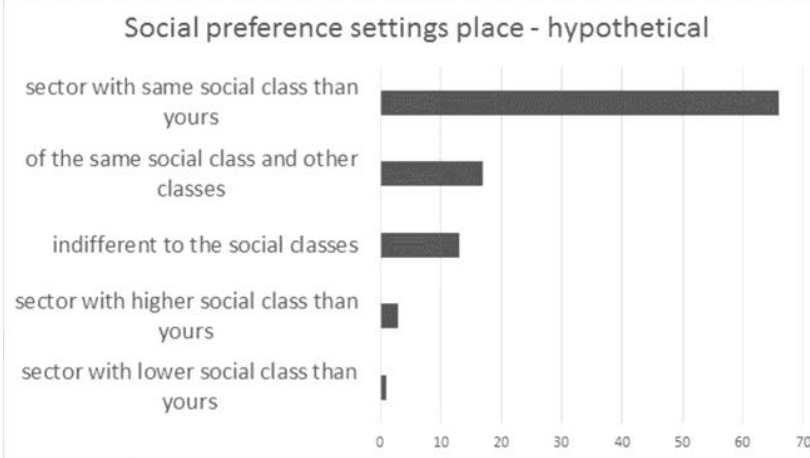

Gráfico 5 Preferencias de localización hipotéticas Fte.: Elaboración propia

Parece que existe una brecha significativa entre la gente de diferentes estratos en función de su experiencia cotidiana, en la medida en que utilizan diferentes espacios. En general, las premisas se asocian con la homogeneidad, la diversidad se asocia principalmente con los sitios de alojamiento de usos comerciales y espacios públicos donde hay más control (o reglas explícitas o implícitas). Por lo tanto, la principal fuente de experiencia que tenemos de otra de diferente nivel socioeconómico es principalmente en espacios públicos o comerciales o en el 
transporte público, pero la ausencia de normas explícitas que genera una incapacidad para predecir el comportamiento impide la generación del enlace.

En los espacios públicos, la sensación principal asociado con la presencia del otro es incómodo. Este malestar se asocia con la inconmensurabilidad de la diferencia entre las clases sociales, esto pone en duda la posibilidad de crear un vínculo entre iguales. Desde la mirada de los entrevistados, este malestar da lugar a conflictos sociales que puedan surgir en la ausencia de expectativas recíprocas de comportamiento. Este malestar se produce en paralelo a un proceso de naturalización de la alteridad (Saravi, 2008), en el que la actitud natural a la otra se produce en el contexto de la indiferencia. De esta manera, el otro va a formar un elemento del medio ambiente con el que no existe la obligación de interactuar. Al evitar la interacción a través de la indiferencia, la sensación de malestar asociada con el comportamiento impredecible de otro se resuelve. Por otro lado, hay espacios donde el estado de incomodidad desaparece porque las expectativas son reguladas.

El resultado de esta fase fue el desarrollo de indicadores que se puede medir cuantitativamente en la segunda fase de la investigación que se describe a continuación.

\subsection{FASE 2 Estudios cuantitativos - Configuraciones de espacios públicos}

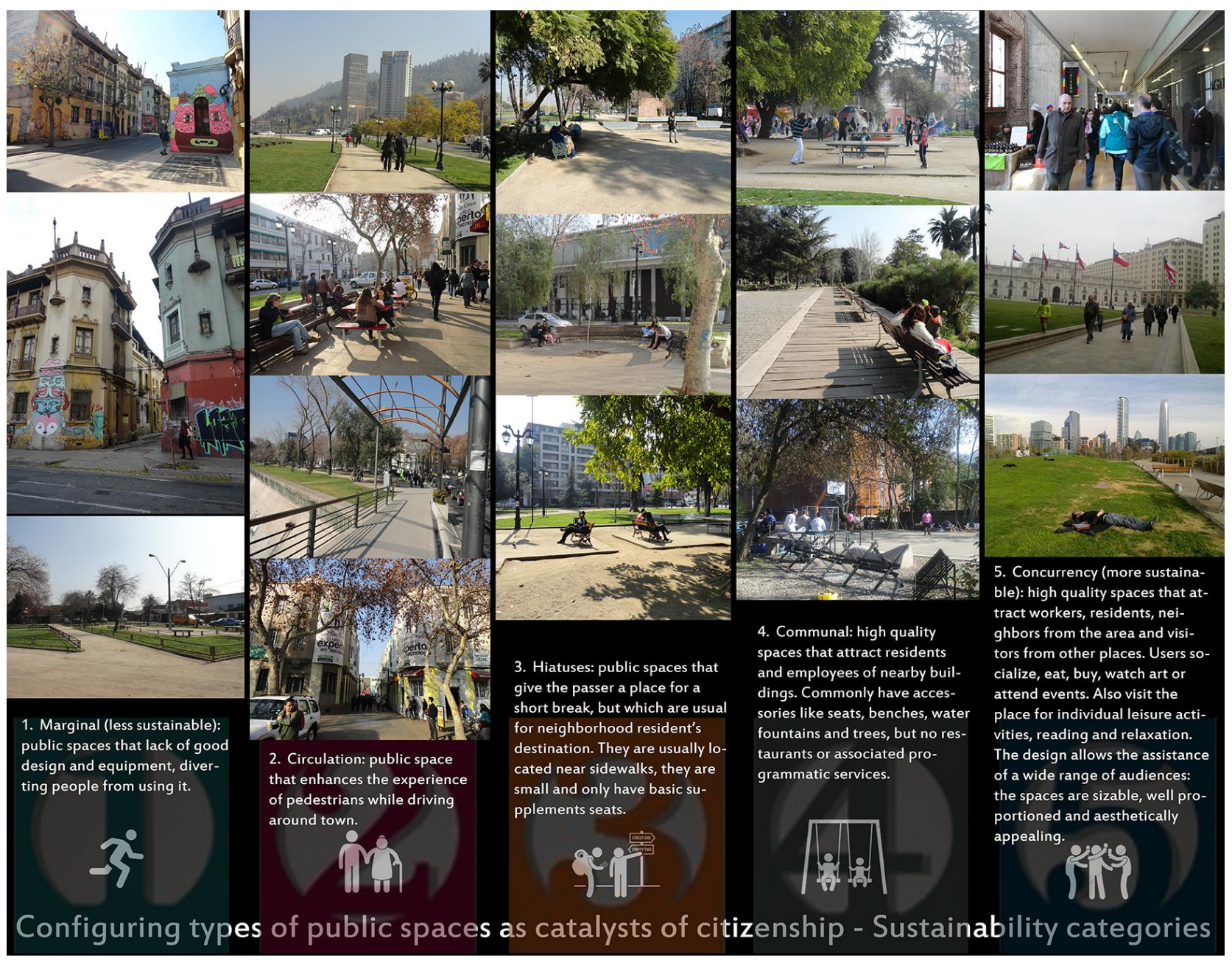

Fig. 2 Configuración de espacios públicos como catalizadores de ciudadanía

Fte.: Elaboración propia 
A través de entrevistas informales se detectó la desigualdad social como uno de los mayores desafíos para el que cruza la ciudad de Santiago, lo que conduce a la delincuencia y la inseguridad. La creación de una tipología de los espacios públicos selecciona tipos apropiados de respuesta a las necesidades de cobertura territorial, cuantitativa, cualitativa y funcional, teniendo en cuenta la diversidad de situaciones existentes. Esto facilita la determinación de las variables de diseño y modelo de gestión adecuada, la optimización y mejora de los componentes físicos y espaciales, integración urbana y mejor ajuste social, que contribuyen a la sostenibilidad general del espacio público resultante. La diferenciación tipológica de los espacios públicos se realiza por función, tamaño y características de diseño predominantes. La definición del tipo de intervención y la definición tipológica de los espacios públicos, son muy importantes en el contexto de las decisiones de planificación durante la fase inicial de la gestión de los espacios públicos.

Las funciones desempeñadas por los espacios públicos se pueden definir en el camino a la vida de la ciudad en cinco categorías por espacios menos sostenibles (aquellos que se convierten en espacios residuales) a espacios más sostenibles (los que están llenos de actividades y continuamente personas) (Fig. 2).

- Marginales: espacios públicos que carecen de un buen diseño y equipo, desviando a que las personas lo usen.

- Espacios intersticiales. Espacios adyacentes que invitan abandono.

- Espacios remanentes. No tiene ninguna función en particular, carecen de identidad, donde el usuario no se identifica $y$, en consecuencia quedan marginados.

- Circulación: el espacio público que mejora la experiencia de los peatones mientras se conduce por la ciudad.

- Aceras. Son espacios que delimitan, áreas privadas y áreas públicas. Son las circulaciones que están dando flujo peatonal a toda la ciudad.

- Espacios de transición. Funcionan como una red para moverse del punto A al punto. Puede albergar un programa de encuentro y de ocio.

- Paseo. Funcionan como áreas de recreación, constituyendo redes capaces de conectar espacios con diferentes tipologías, para deporte o contemplación.

- Comercial. Va en diversidad de escalas, en tanto se produce la interacción entre las personas y éstas se relacionan en función de sus intereses y / o necesidades.

- Hiatos: espacios públicos que dan al transeúnte un lugar para un breve descanso.

- Plazoletas. Puntos de encuentro para personas que diariamente pasan. Espacio de transición, el mobiliario no es esencial, pero casi todos tienen.

- Retranqueos. Alejamiento total o parcial de una fachada en la planta baja de los edificios. Crean espacios públicos permitiendo a que los edificios se adapten al contexto.

- Jardines centrales de carreteras. Sirven como espacios de transición mientras que una barrera de seguridad. A menudo se crean para generar una imagen de una ciudad saludable y un ambiente acogedor.

- Comunitario: atraen habitantes de áreas cercanas. Comúnmente tienen accesorios (bancos, fuentes de agua, árboles), pero no servicios programáticos asociados. 
- Parque comunitario. Lugar de encuentro para los residentes de la zona y lo utilizan también como un punto de referencia.

- Espacio pseudo-público. Espacio público en manos privadas. Las configuraciones físicas están determinadas por las características del mercado y el área de influencia: cerrado, abierto e híbridos.

- Áreas deportivas. Son de los pocos espacios que pueden generar economía. Pueden ser cerrados o abiertos como y mayormente respetan las escalas de la comunidad y atraen ciudadanos de diferentes clases sociales.

- Concurrencia: atraen personas de la zona y visitantes de otros lugares. Adicional a accesorios cuenta con programas y servicios complementarios permitiendo una amplia gama de audiencias.

- Parque intercomunal / metropolitano. Une varias comunidades. Se da a la interacción de un gran grupo de personas de diferentes clases sociales.

- Zona turística. Sirvieron como un símbolo de la ciudad. Convergen diversos tipos de personas: los ciudadanos locales, turistas nacionales, turistas extranjeros y los que trabajan en la zona.

- Jardines. Espacio para el descanso, la relajación, la meditación y el contacto con la naturaleza, además para un enfoque más práctico, como la producción de una variedad de vegetación la educación y turismo.

- Plaza. Espacios rodeados de zonas verdes, totalmente desprovistas de elementos naturales y techos. En el que una variedad de actividades están integradas. El significado de estos espacios va cambiando con el tiempo.

Se puede decir que los espacios públicos de concurrencia son clave para alinear los intereses económicos del sector privado y de los intereses sociales y ambientales del sector público. Teniendo en cuenta entonces que la sostenibilidad es el punto clave en el éxito de los espacios públicos y su duración en el tiempo (Proyecto para Espacios Públicos). Entre estos se encuentran primeramente los accesos y enlaces, la relación entre el sitio y su entorno, tanto físico como visual. Visible desde lejos, para que las personas que se interesen en entrar. Debe ser accesible a pie y cerca del transporte público. Las empresas son importantes sobre todo tiendas. Segundo, la imagen y comodidad. El concepto de comodidad consiste en la sensación de seguridad, la limpieza y la disponibilidad de asientos con diferentes cualidades (sol y sombra, escaleras, bancos, etc.). Tercero, el uso y actividades. Las actividades son los elementos básicos de un lugar, dar a la gente una razón para usarlos y volver en el futuro. Cuando no hay nada que hacer, o no es adecuado para espacios de recreación, por lo general el espacio queda vacío. Cuantas más actividades que acojan el sitio, se dará a más personas la oportunidad de participar en ellos. Como último punto, la socialización. Una cualidad difícil de conseguir en un lugar, pero es cuando las personas se reúnen para ver a los amigos, conocer o saludar a los vecinos, o incluso sentirse cómodo interactuando con extraños. Hay un sentido de propiedad del lugar.

\section{Conclusión}

Con relativa independencia de su nivel social, los residentes de Santiago, muestran una disposición significativa a la integración de espacios públicos con personas de diferentes condiciones sociales. Sin embargo, por un lado, esta evaluación positiva de la integración enfrenta obstáculos concretos y temores que hacen más difícil la materialización de los espacios públicos menos segregados. Se pueden relevar tres factores que tienen impacto. El 
primero es la estructura de oportunidades, oportunidades efectivas para cumplir con otros en diferentes zonas de la ciudad. Algunas características físicas: escala, la penetración de los servicios privados, experiencia de movilidad. Las características estructurales definen el tipo de oportunidades para que los diferentes grupos socioeconómicos puedan elegir, lo que resulta en más espacios homogéneos o heterogéneos. Segundo, el significado cultural que los sujetos hacen de estas oportunidades y contactos de la permeabilidad con la otra, teniendo en cuenta el diferente como un igual y no bajo las relaciones jerárquicas. Los patrones culturales proporcionan legitimidad a la distribución de oportunidades, dan sentido a estas oportunidades y generan marcos de interpretación, evaluación y comportamiento en las relaciones con personas de otros estratos. Finalmente, la historia y características sociales y físicas de cada influencia de la ciudad en la apertura al "otro" diferente. La combinación de ambas dimensiones se traduce en ciertos niveles de contacto entre personas de diferente nivel socioeconómico y ciertos espacios físicos de contacto entre diferentes.

Los sectores más homogéneos de la ciudad en el caso de Santiago, corresponden a grandes aglomeraciones de la pobreza, como el noroeste y suroeste de la ciudad, no en los suburbios de altos ingresos. El sector conocido como "parte alta", aunque se concentra en la mayoría de las familias de ingresos altos de la ciudad, es permeable a otros grupos sociales, lo que hace que sea mucho más heterogéneo que los pobres. Es por esto que se concluye que las distinciones que la gente hace, no son totalmente alineadas con los ingresos, sino por elementos transversales como el esfuerzo, el territorio, etc. El mayor factor de distinción es sociocultural y no son necesariamente económicas, posibilidades de mezcla espaciales están abiertas en términos socioeconómicos. Es entonces, cuando el espacio público vuelve a aparecer en el discurso urbano, como la oportunidad de reconstruir la solidaridad urbana. La forma específica de la tolerancia entre lo social, mezcla de empatía, de indiferencia y el miedo, parece un fenómeno de la atmósfera psicológica de las ciudades (Simmel, 1988). La indiferencia y el anonimato, lejos de ser negativo, son los pilares de la vida pública (Sennett, 1977).

En síntesis, aun existiendo una convivencia deliberada entre las diferentes clases sociales, en los discursos de los entrevistados aparecen algunas condiciones que permiten a dicha coexistencia. Por lo tanto, lo que está ocurriendo a nivel cultural es la formación de un núcleo de valores que legitima la distribución de las oportunidades sociales ( $y$, por tanto, su traducción en las zonas con ciertas características) del esfuerzo individual y dentro de un escenario de igualdad de oportunidades. Lo que parece problema no es la segmentación socioeconómica de espacios, pero la desigualdad de oportunidades. La heterogeneidad social de los espacios no es visto como un fin en sí mismo. La historia emerge con fuerza la falta de vínculo efectivo con otro de diferente nivel socioeconómico. Este debilitamiento del vínculo tiene consecuencias importantes asociados con la valoración de los demás. En la medida en que la experiencia cotidiana de la otra se debilita, lo que se necesita para establecer la alteridad es un imaginario permeado por la desconfianza y la ignorancia del otro. Se basa en este imaginario, que la disposición se decide a la participación en zonas socialmente mezcladas.

\section{Referencias}

Andara, E. (2009). La Formación del Espacio Público en América Latina. Anuario GRHIAL Nº 3, Universidad de Los Andes, Carácas.

Barozet, E., Fierro, J. (2015). Clase media en Chile: Implicancias sociales y políticas. Revista Paraguaya de Sociología.

Mollenkopf, J. H. \& Castells, M (1991). Dual city: Restructuring New York. Nueva York: Russel Sage Foundation. 
Doxiadis, C.A. (1968). Ekistics: An Introduction to the Science of Human Settlements. New York: Oxford University Press.

Goodsell, C. (2003), The concept of public space and its democratic manifestations, en The American Review of Public Administration, Diciembre $N^{\circ} 33$ 361-383, University of Missouri, Missouri.

Güell, P. (2005). Desarrollo Humano y Ciudadanía en Chile, Recuperado en Diciembre 6, 2009, de: www.revistapolis.cl/polis final/12/guell.htm

Habermas, J. (1989), El discurso filosófico de la modernidad. Doce lecciones, Taurus, Barcelona.

Hopenhayn, M. (2007). Derechos sociales, deudas pendientes y necesidad de un nuevo pacto social. Notas de población, 85, 15-30.

Kayden J. (2000). Privately Owned Public Space: The New York City Experience.

Lindon, A. (2007). La ciudad y la vida urbana a través de los imaginarios urbanos. EURE (Santiago), Vol.33. n॰99, pp.7-16.

Lindon, A. (2007). La construcción social de los paisajes invisibles del miedo. In: Joan, Nogué (ed.) La construcción social del paisaje. pp. 219-242. Madrid: editorial biblioteca nueva S.L.

Mehran N. (2013). Frontiers of Business, Management and Economics: An Interdisciplinary Collection of Managerial Research Findings and Breakthroughs, Universal-Publishers.

Sabatini, F; (2009). Disposición a la Integración Social Urbana en Tres Ciudades de Chile (Santiago, Antofagasta, Temuco): Una Mirada desde la Integración Residencial. MINVU, ProUrbana UC and OSUAH.

Marquez, F. (2007). Imaginarios urbanos en el Gran Santiago: huellas de una metamorfosis. Eure. 99(33): 79-88. ISSN 0717-6236.

Sabatini, F; Wormald, G; Trebilcock, M. y Rasse, A. (2013) Cultura de Cohesión e Integración Social en Ciudades Chilenas. Editores Colección Estudios Urbanos UC.

Saravi, G. (2008) Mundos aislados: segregación urbana y desigualdad en la Ciudad de México. Eure. 34(113): 93-110, ISSN 0717-6236.

Sennett R.; Harvey C. (1977). The Fall of Public Man

Simmel, G. (1988 [1903]). La metrópolis y la vida mental. En M. Bassols, R. Donoso, A. Massolo \& A. Méndez (Comps.), Antología de Sociología Urbana (pp. 47-61). México: Universidad Nacional Autónoma de México (UNAM).

Simmel, G. (2000 [1918]) El conflicto de la cultura moderna. Revista Española de Investigadores Sociológicas, 89,315-330.

Torche, F. y Wormald, G. (2004). Estratificación y movilidad social en Chile: entre la adscripción y el logro. Santiago, Chile, CEPAL, División de Desarrollo Social. 85 p. ISBN 92-1-322604-7.

Torche, F. (2005) Unequal but fluid: social mobility in Chile in comparative perspective. American Sociological Review. 70(3): 422-450, ISSN 00031224. 